\title{
L'histoire, ça sert à faire la guerre
}

History Serves to Make War

\section{Yves Ansel}

\section{OpenEdition}

Journals

Édition électronique

URL : http://journals.openedition.org/recherchestravaux/888

ISSN : 1969-6434

Éditeur

UGA Éditions/Université Grenoble Alpes

Édition imprimée

ISBN : 978-2-37747-006-8

ISSN : 0151-1874

\section{Référence électronique}

Yves Ansel, «L'histoire, ça sert à faire la guerre », Recherches \& Travaux [En ligne], 90 | 2017, mis en ligne le 15 juin 2017, consulté le 08 septembre 2020. URL : http://journals.openedition.org/ recherchestravaux/888

Ce document a été généré automatiquement le 8 septembre 2020

(c) Recherches \& Travaux 


\title{
L'histoire, ça sert à faire la guerre
}

\author{
History Serves to Make War
}

Yves Ansel

\section{Entre Stendhal et l'histoire, une longue histoire d'amour}

1 Comme le prouve la patiente enquête de Victor Del Litto portant sur la généalogie des idées de Stendhal ${ }^{1}$, entre l'auteur de la Vie de Napoléon et l'histoire, c'est une longue histoire d'amour. C'est très tôt que Beyle voit dans l'histoire une « chose principale » et "précieuse dans ce siècle ${ }^{2}$ », adore Saint-Simon ${ }^{3}$, goûte les correspondances privées et les mémoires, ouvrages dont il «a été un lecteur constant et passionné ${ }^{4}$ ». Même si le Grenoblois monté à Paris pense que son " true talent » est celui de «comic bard" ", dans les multiples projets (pas toujours "ciblés », et sans suite effective) qu'il jette sur le papier, il n'omet guère de songer à « programmer » des ouvrages historiques:

Histoire de Bonaparte

Histoire de la Révolution française.

Histoire des grands hommes qui ont vécu pendant la Révolution française.

Commencer ces 3 ouvrages à 35 ans, dans 15 ans d'ici. ${ }^{6}$

2 Notable «petit fait vrai » : le premier livre d'un volume conséquent signé par le futur écrivain est un ouvrage historique, rédigé en $1808^{7}$. À cette époque, "non seulement Stendhal fait à Brunswick sa lecture préférée des livres d'histoire, mais encore il devient lui-même historien ${ }^{8} »$. Victor Del Litto constate la chose, sans plus. Dans sa «Grande Biographie », Michel Crouzet n'est guère plus prolixe ni précis : « Tout près de Brunswick se trouve, à Wolfenbüttel, une célèbre bibliothèque qu'avait dirigée Leibnitz. Stendhal y emprunte des livres, en particulier pour cette Histoire de la guerre de Succession d'Espagne, entamée en 1808 [...] $]^{9}$. ' C'est l'article, fondateur, essentiel, de Gérald Rannaud, "Stendhal et la tentation de l'histoire ${ }^{10}$ ", qui a sorti de l'ombre cet 
essai, et permis d'en mesurer toute la longue portée. Dans son éclairante analyse, Gérald Rannaud fait pertinemment remarquer deux choses :

- que «le premier travail littéraire auquel notre apprenti dramaturge soit parvenu à donner une certaine ampleur [" plus de trois cents pages »] est, en 1808, un ouvrage historique »;

- que l'ouvrage est un texte polémique codé, qui fait de l'histoire un usage intéressé, politique. En la circonstance, censure impériale oblige, l'intendant aux ordres de Daru use de « la voie oblique » (Victor Brombert) pour désavouer les visées annexionnistes de Napoléon (l'entrée en guerre avec l'Espagne). Sous couvert de parler d'une autre guerre traitant des démêlés de Louis XIV avec la couronne espagnole, Beyle critique à mots couverts les ambitions territoriales de «son » Empereur.

Que la portée polémique de cette Histoire soit à ce point hypervoilée que le sens second du texte n'apparaisse pas assez nettement, c'est indéniable. Une chose du moins est sûre: une guerre sert de couverture à une autre, un texte (le récit de la guerre d'Espagne) en cache un autre (la critique de l'annexionniste politique napoléonienne), mais en l'occurrence le sous-texte est trop immergé, trop profondément enfoui pour que le but du factum soit atteint: la cible frappée par ricochet (Napoléon) est trop dissimulée, l'attaque trop " entre les lignes » (une « erreur d'encodage » qui s'explique fort bien: au service de l'Empereur, tenu au secret et à l'obéissance, l'Intendant prudent a tellement codé son Histoire que celle-ci n'est pas vraiment décodable !) pour que la charge contenue dans l'essai soit clairement lisible. De ce point de vue, et pour les mêmes raisons qu'Armance (roman où Stendhal a également surdosé le taux de censure, ce qui rend ipso facto le récit opaque, incompréhensible), cette History of the War of Succession est ratée. Quoi qu'il en soit, la stratégie mise en œuvre, testée dans cette première Histoire, sera reprise ensuite (avec plus de talent) dans tous les autres ouvrages (historiques ou non), tous plus ou moins ouvertement engagés. Une fois libéré de ses chaînes officielles (après la chute de Napoléon en avril 1814), hors service, quand Stendhal peut écrire, certes non sans garder un œil sur ces "MM. de la police», non sans devoir s'autocensurer ${ }^{11}$, mais sans l'impératif catégorique de s'avancer aussi masqué, quand il y a objectivement pour lui une relative levée de censure, alors apparaît clairement quel usage indirect, pragmatique et polémique l'écrivain fait de l'histoire (une couverture, un alibi, un " paratonnerre " pour parler du temps présent) - un usage déjà en place, mais non encore rôdé, et trop empêtré (parce qu'« enchaîné dans [l'] expression $\left.{ }^{12} »\right)$, passablement embrouillé, dans l'essai de 1808.

\section{Napoléon en 1812 : la prise de conscience historique}

Dans la genèse de la pensée beyliste, si l'intérêt pour l'histoire est pour ainsi dire originel, l'importance prise par celle-ci dans les écrits de la maturité ne s'explique nullement par ce précoce et indéfectible investissement intellectuel. Stendhal s'est à ce point passionné pour l'histoire parce que l'Histoire (comme chance, destin et cauchemar) lui est tombée dessus, parce que c'est toute la vie de Beyle qui, bon gré mal gré, s'est trouvée prise dans les remous du tumultueux cours de la grande Histoire. Sans accréditer les bévues beuviennes ("Tel arbre tel fruit», tel homme telle œuvre) pour expliquer la vision du monde de Dominique, on ne saurait - autre bévue méthodologique - faire totalement l'impasse sur « le vécu » de Beyle, faire abstraction d'une vie d'homme aussi étroitement en phase avec l'histoire en train de se faire. De fait, il se trouve que les dates qui comptent dans la vie de Henri Beyle (à commencer 
par l'arrivée à Paris quelques jours après le 18 Brumaire) sont aussi des dates historiques, que l'histoire personnelle est enchâssée dans la grande Histoire, imbriquée dans les conquêtes et guerres de Napoléon, embarquée dans les orages politiques qui marquent le début du siècle. « Né en 1783, dragon en 1800, étudiant de 1803 à $1806^{13}$ ", au service de l'Empereur de 1806 à 1814, Beyle «tombe » avec Napoléon. Démobilisé, demi-solde devenu indésirable après 1815 , il part pour l'Italie d'où la politique (libéral soupçonné de carbonarisme, Beyle est forcé de prestement quitter Milan) le chasse en 1821. Retraité politique ne pouvant trouver d'emploi en France, Beyle vit en écrivant, en imprimant. C'est un autre changement de régime (la révolution de 1830) qui modifie de nouveau le cours de sa vie, lui donne un nouvel emploi, lui octroie un poste de consul à Trieste (où, persona non grata, il est prié de déguerpir), puis à Civitavecchia. Ces dates et césures "parlent haut et fort "(Balzac). Jamais avant Henri Beyle, une vie d'écrivain n'avait été à ce point marquée par la politique, par le poids et l'accélération soudaine de l'histoire, et c'est précisément ce dont rend compte à sa manière la Vie de Henry Brulard, cette singulière autobiographie (la première du genre) qui prouve à quel point le moi est un produit familial et social, une conscience sujette, intimement dépendante de l'histoire qui façonne l'individu.

Que l'homme soit un " animal historique », cette idée en voie d'apparition, Beyle ne l'a certes pas apprise dans les livres (qui postulent une "nature humaine " stable, qui ne parlent que d'un Homme invariable, intemporel, identique à lui-même depuis toujours), il l'a apprise au cours de ses voyages et campagnes dans le sillage de la Grande Armée, et, surtout, en 1812.

Dans la vie vagabonde et mouvementée de Beyle, il y a cette année-là : « 1812 : Moscou ${ }^{14}$ ».

7 De la campagne de Russie, Beyle a un peu parlé (dans sa correspondance, son journal, ses conversations), Stendhal n'a dit mot. Non que 1812 n'ait durablement et profondément marqué le futur écrivain, loin s'en faut. Beyle (innocent, «ignorant comme une carpe » au sortir d'une enfance surprotégée) sait que ce voyage au bout de l'enfer l'a déniaisé, l'a affranchi (la guerre vue de près lui fait perdre ses illusions rousseauistes : l'homme est un prédateur, un animal sauvage, un loup pour l'homme), a fait de lui un autre homme. Mais l'écrivain élude, censure, refoule 1812. Parce qu'il n'y a pas de mots pour le dire? Par honnêteté, pudeur, modestie? Par noblesse d'âme? Parce que ce qu'il a vu « là-bas » est indicible et inaudible ${ }^{15}$ ? Sans doute pour toutes ces raisons, mais si les écrits escamotent cette " chose terrible ${ }^{16}$ ", l'année 1812, elle hante les écrits de Stendhal, rôde et s'immisce dans les chronologies (politiques, historiques, artistiques), revient par la bande là où elle n'est pas toujours attendue, objectivement à sa place, parce que, aux yeux de l'écrivain, elle est une date clé tant dans l'histoire politique de l'Europe moderne que dans les arts et la littérature ${ }^{17}$.

\section{Stendhal lecteur de la nouvelle école historique}

Tout en croyant fermement que son «true talent » est celui de « comic bard », Dominique entre toutefois en littérature par l'histoire. À l'essai de 1808 font suite les Vies de Haydn, de Mozart et de Métastase (1814), l'Histoire de la peinture en Italie (1817), la Vie de Napoléon (mise en chantier à Milan en 1817-1818) ainsi que la Vie de Rossini (1823), des Vies qui, dans l'esprit de l'auteur, relèvent pleinement de l'histoire: "J'ai écrit les vies de plusieurs grands hommes: Mozart, Rossini, Michel-Ange, Léonard de Vinci. Ce fut le 
genre de travail qui m'amusa le plus. Je n'ai plus la patience de chercher des matériaux, de peser des témoignages contradictoires, etc.; il me vient l'idée d'écrire une vie dont je connais fort bien tous les accidents. Malheureusement, l'individu est bien inconnu: c'est moi ${ }^{18}$.»

Cette primauté et antériorité de l'histoire sont d'une conséquence capitale non seulement parce que le discours historique va substantiellement nourrir tous les écrits ultérieurs de Stendhal mais aussi parce que c'est en amateur éclairé, en connaisseur, que le journaliste (de 1822 à 1829) chargé de rendre compte de l'actualité littéraire en France sous la Restauration - un âge d'or pour la littérature historique, une époque marquée par le phénoménal succès des romans de Walter Scott, des Mémoires et de l'histoire - découvre, au fil des ans et dans le cadre même de son métier (chroniqueur culturel), les multiples productions de ceux qui, a posteriori, vont apparaître comme les promoteurs d'une nouvelle école historique: François Guizot (1787-1874), Augustin Thierry (1795-1856), François Mignet (1796-1884), Adolphe Thiers (1797-1877), Jules Michelet (1798-1874), Edgar Quinet (1803-1875).

$\mathrm{Au}$ delà des différences d'appréciations résultant d'approches mettant l'accent plutôt sur telle ou telle facette des bouleversements révolutionnaires, tous estiment que l'histoire a un sens et va dans le bon sens. Parce que tous ces historiens " appartiennent, par leurs origines et par leur idéologie, à la classe bourgeoise et à ses différentes nuances ${ }^{19}$ ", ils voient dans le temps un progrès et dans la Révolution un grandiose événement positif. De retour à Paris en 1821 et devenu journaliste (pour des revues anglaises) à des fins alimentaires (il faut vivre ${ }^{20}$ ), Stendhal est professionnellement tenu de lire les nouvelles parutions, et bien que depuis longtemps passionné par l'histoire, l'écrivain est visiblement passé à côté de ce que les historiens novateurs de la période ont pu apporter, découvrir. Rien d'étonnant à cela :

- par définition, le chroniqueur écrit dans l'urgence, ne dispose d'aucun recul. Il livre à la chaîne des comptes rendus ponctuels, sans chercher plus avant ni faire retour sur ce qu'il peut lire, sans souci (et possibilité) de synthèse, sans avoir non plus à se poser la question de savoir ce que ces historiens peuvent avoir en commun, révéler ou cacher.

- Stendhal lit Guizot, Barante, Augustin Thierry ou Thiers avec des yeux informés, et prévenus. Le journaliste n'est nullement un plumitif novice ; il a déjà son idée sur l'histoire, il a son opinion forgée, et bien arrêtée, sur la Révolution ou Napoléon (une période héroïque à laquelle il a été mêlé et sur laquelle il a médité, écrit), et, de surcroît, il a, prête à l'emploi, sa conception du devenir historique, et une conception singulière, agnostique (Stendhal ne croit pas aux lendemains qui chantent : attribuer un sens à l'histoire, c'est croire à la fable d'un paradis au bout de la route du progrès), qui ne le prédispose guère à partager (et donc à voir et louer) celle véhiculée par Guizot, Thiers et tutti quanti. À la différence des historiens de «la génération 1820 » en effet, Stendhal ne pense pas que l'histoire ait un sens évident, encore moins qu'elle aille nécessairement dans le bon sens. L'évolution historique n'est ni une ni unilinéaire, si bien que la durée ne peut se lire sur une seule portée (de la tyrannie monarchique à la liberté et à l'égalité des droits de l'homme, par exemple). Le temps historique est complexe, fort emmêlé, à la fois positif et négatif parce qu'il entrecroise de multiples temporalités décalées, désaccordées et irréductibles. L'interprétation du passé (qui est toujours une construction) dépend du point de vue adopté, des tables des valeurs retenues : ce que l'on gagne sur certains tableaux on le perd sur d'autres ${ }^{21}$.

- À l'heure où le discours historique commence à se professionnaliser, Stendhal en reste à l'idée d'une histoire qui doit servir à éclairer les esprits, intervenir dans les débats de la cité. 
- Stendhal lit ces historiens non comme s'ils étaient des pairs, non comme les lirait un véritable historien, mais comme homme de lettres engagé dans les débats de son temps, et comme écrivain. Ce qui lui importe le plus, ce qui retient le plus son attention dans toutes ces histoires, ce ne sont pas les critères des professionnels, des historiens de métier. Le travail sur les archives, l'exploration et exploitation de nouveaux matériaux, l'exactitude de la peinture, le respect de la vérité historique, etc., tout cela est certes admirable, mais secondaire à ses yeux. Rien ne sert d'être un savant hors pair, un historien compétent si l'ouvrage final est froid (id est sans passion et sans relation avec les préoccupations actuelles, les questions du moment), pesant, pédant, ennuyeux. Dans la littérature historique comme ailleurs, il faut séduire, intéresser, plaire, amuser.

11 Tout compte fait, Stendhal n'a pas tiré grand profit de ces lectures. C'est qu'il a découvert ces historiens, dont il reconnaît et loue la valeur, quand sa pensée était mûre, quand il est trop tard pour que la nouvelle historiographie modifie sensiblement sa façon de voir, change son fusil historique d'épaule. L'auteur passionné de Vies continue d'adorer sans réserves Saint-Simon (un «historien» amateur d'anecdotes futiles dont il ne perçoit nullement les limites), de privilégier indûment Grands et "grands hommes", de surestimer l'importance du nez de Cléopâtre (petits faits, grands effets), des idées, des salons et des élites, de sous-estimer totalement le peuple, l'économie, le commerce, les réalités prosaïques, etc.

\section{«Un passé-arsenal22 »}

12 Après un tir groupé d'écrits historiques, sans délaisser pour autant l'histoire (l'écrivain entreprend encore deux «Vies » inachevées : la Vie de Henry Brulard en 1835, et les Mémoires sur Napoléon en 1836-1837), Stendhal s'engage dans d'autres voies, explore d'autres genres (récits de voyages, pamphlets, essai, chroniques journalistiques, fictions). Reste que, dans la chronologie des œuvres de l'écrivain polygraphe, c'est bien l'historien qui est premier, et c'est l'historien qui fait de Beyle un écrivain. Un constat dont il importe de prendre toute la mesure (le roman réaliste sera, par définition, un roman historique). C'est fort justement que, dans sa thèse, Xavier Bourdenet, consacre tout un chapitre à "Stendhal historien ${ }^{23}$ " et parle de "décade historique " (de 1810 à 1820) pour caractériser la teneur et le genre des premiers projets et premières publications ${ }^{24}$.

13 Cela clairement posé, pas plus qu'on ne saurait enfermer le polémiste dans ce qui apparaîtrait comme un «septennat pamphlétaire»(de 1818 à 1825 : Des périls de la langue italienne...à Racine et Shakespeare II), pas plus que l'activité du libelliste ne se réduit aux trois pamphlets publiés parce que « la parole pamphlétaire » (Marc Angenot) est consubstantielle aux « œuvres complètes » de l'écrivain ${ }^{25}$, le Stendhal historien n'est nullement confiné dans les écrits relevant expressément de genres historiques dûment attestés. Chez Beyle et Stendhal, « la parole historique » (pour reprendre et adapter ici l'expression de Marc Angenot) est absolument partout, et du début (l'essai de 1808) à la fin (Idées italiennes sur quelques tableaux célèbres, 1840). Et cette diffuse "parole historique » est, naturellement et constamment, au service de «la parole pamphlétaire ».

14 Ainsi que l'avaient fait Bayle (Dictionnaire historique et critique) et Voltaire (Essai sur les mœurs, Dictionnaire philosophique), et comme devait le faire délibérément et systématiquement Karl Marx, ou encore le Barthes des Mythologies (dans la mesure où le 
mythe " transforme l'histoire en nature ", l'histoire est par essence " dénaturalisante ", démystificatrice), Stendhal use stratégiquement de l'histoire (magasin de grands et "petits faits vrais ", répertoire d'exemples concrets frappants, parlants, irréfutables) contre l'idéaliste philosophie pérenne (considérée en bloc comme un ensemble de "divagations » et de "rêveries", comme une sempiternelle, verbeuse et ratiocinante "fable »), pour penser l'homme réel (dans son siècle, sa société, son "outillage mental»), miner les discours reçus, et passer à l'attaque. Stendhal historien? Assurément non, si l'on entend par le mot « historien » un expert patenté ès choses du passé qui se plonge dans les archives, qui passe au crible les différentes sources d'informations, et veille à livrer une histoire informée, exacte, raisonnée, aussi dépassionnée que possible, des époques, des domaines, des thèmes et objets qu'il entend explorer ou éclairer. Stendhal n'a rien à voir avec cet historien-là. Mais c'est parce que l'histoire, pour lui, n'a rien à voir avec un savoir, avec l'histoire des " antiquaires » et des «savants » (peu ou prou toujours " pédants » dans le lexique de Beyle ${ }^{26}$ ). Pour Stendhal, l'histoire est une banque de données utiles, un arsenal d'actions, de grands hommes, de situations, de scénarios, de valeurs, de mentalités, etc., un stock d'armes offensives susceptibles de fournir des arguments, des preuves au service des idées, des thèses de l'écrivain.

Bref, pour Stendhal, l'histoire, ça sert à faire la guerre.

La place me manque ici pour entrer dans le détail des usages et finalités du passé dans les textes stendhaliens, et je me limiterai donc à évoquer seulement l'utilisation que fait le pamphlétaire des événements des dernières décennies dans la défense et illustration $\mathrm{du}$ romantisme ${ }^{27}$. L'histoire en effet est l'argument massue du champion de Shakespeare, du "moderne » contre les "classiques». " Tout est histoire», cela veut dire que, loin d'être un long fleuve tranquille, une année chassant l'autre selon le défilé des jours et l'ordre des saisons, la durée historique est une succession de catastrophes, de ruptures, de discontinuités, qui font de l'homme une création de l'homme, un mutant constant, un être qui ne se ressemble pas d'une époque, d'un siècle, d'une culture à l'autre. Le romantisme de Stendhal est étroitement lié à la découverte d'une histoire faite de déchirures, de changements radicaux qui transforment les hommes et périment les civilisations et arts d'un autre âge. Les chefs-d'œuvre du siècle de Louis XIV ont fait leur temps, sont désormais obsolètes parce que les Français de 1823 ou 1825 n'ont plus rien à voir avec les Français de 1670. À des temps nouveaux il faut une nouvelle littérature. Ce qui vaut pour Racine et Molière (auteurs du siècle de Louis XIV, désormais déclassés, périmés) vaut a fortiori pour les littératures antédiluviennes. Au rebours de toute la Tradition - et ce parce qu'il a une inédite et claire conscience de l'historicité de l'humanité - , Stendhal pense que l'homme du XIX siècle n'a plus rien de commun avec les Grecs ou les Romains, tout simplement parce que la société, les mœurs et valeurs de ces peuples-là sont morts avec eux, parce que la "chaîne des temps" est une formule creuse (ladite "chaîne" a été maintes fois rompue), parce que les pensées et mentalités ont changé du tout au tout au cours des siècles, tant et si bien que les Anciens sont pour nous de parfaits inconnus ${ }^{28}:$ «Le public français ne sympathise plus aux malheurs d'Agamemnon et d'Iphigénie; de tels héros et héroïnes sont trop éloignés de nous ${ }^{29}$. " Sauf à ignorer les faits vrais ou à philosopher sur les hauteurs, dans les nuées métaphysiques qui font s'évaporer la vie matérielle, disparaître détails têtus, évolutions et révolutions, l'histoire oblige à 
constater et conclure que l'Homme n'existe pas, partant que les époques sont hermétiques, indéchiffrables les unes pour les autres :

Je crains bien qu'en fait de musique, de peinture, de tragédie, ces Français-là [ceux de 1786] et nous [Français de 1825], nous ne soyons à jamais inintelligibles les uns pour les autres ${ }^{30}$.

Et c'est précisément parce que Stendhal tient compte des phases et fractures historiques (l'Antiquité, le Moyen Âge, «le siècle de Léon X », l'instauration du gouvernement monarchique et la montée en puissance de la vanité, la Révolution de 1789 et la démocratie, l'Europe industrielle et le règne de l'argent) que, le premier, il est porté à attaquer de front l'idée que les auteurs et les œuvres classiques (de l'Antiquité ou du XVII ${ }^{\mathrm{e}}$ siècle français) sont "éternels", " de tous les temps comme de tous les lieux ${ }^{31}$ ". Croire en la pérennité des chefs-d'œuvre artistiques, c'est postuler que l'essence précède l'existence, c'est croire en la déclinaison d'une nature humaine éternelle, immuable à travers les âges (credo des "classiques", et credo indissociable d'une théologie).

Où il apparaît clairement que l'histoire, sous la plume de Stendhal, sert efficacement et opportunément les objectifs du journaliste, de l'essayiste ou du libelliste. Et d'une manière générale, l'appel à l'histoire obéit à deux objectifs majeurs :

- Mobiliser le passé pour relativiser le prétendu progrès, minorer les «perfections» des sociétés «civilisées", critiquer les temps modernes, rapetisser le siècle. C'est sans nul doute la fonction la plus récurrente, la plus immédiatement visible - la plus traditionnelle aussi, partant la plus appréciée, glosée et amplifiée par les commentateurs de droite qu'inspirent toujours irrésistiblement la nostalgie et les déplorations attachées à l'idée de décadence - de l'histoire dans les écrits de l'écrivain polygraphe, lequel « vit et perçoit l'histoire sur le mode quasi exclusif de la dégradation ${ }^{32}$ ». Pour évaluer le xIx siècle bourgeois, marchand, industriel, de plus en plus «anglisé », Dominique n'est jamais à court d'adjectifs : au choix, selon le propos, le siècle est «triste » " grave », « plat », « ennuyeux », « sérieux ", " envieux », « empesé », « hypocrite », " raisonneur », etc. Comme l'écrivain ne voit guère l'avenir en rose (l'Amérique ne le fait pas rêver, et si l'Amérique est l'avenir de l'Europe, alors a priori le futur ne vaut décidément pas le passé), l'avenir est bouché ; et même s'il ne fantasme pas non plus sur d'idylliques origines, sur une Arcadie perdue, sur un mythique âge d'or, ses considérations historiques illustrent fort souvent l'antienne : c'était mieux avant.

- Prendre acte que l'homme est un «animal historique », et tirer toutes les conséquences (sociales, politiques, psychologiques, philosophiques, artistiques, éthiques, etc.) de cette idée neuve. Aucune des émotions et réactions humaines n'est purement instinctive, naturelle: amour, ambition, orgueil, violence, jalousie, pudeur, amitié, âme, vanité, peur, tendresse, douleur, etc, la vie affective tout entière (âme comprise ${ }^{33}$ ) porte les stigmates de la société et de l'histoire. Exit l'idée d'une "nature humaine», exit aussi tout ce que cette antique et conservatrice idée-là entraîne à sa suite (des chefs-d'œuvre "immortels » à " l'éternel féminin », infinie est la liste des fictions, des «fables convenues» liées au postulat d'un Homme et d'une Femme immuables, imperméables aux conditionnements sociaux et historiques). Et ce décapant usage-là de l'histoire n'a toujours pas perdu de sa pertinence, de son intérêt, de son actualité... 


\section{NOTES}

1. V. Del Litto, La vie intellectuelle de Stendhal. Genèse et évolution de ses idées (1802-1821), Paris, PUF, 1959.

2. Voir Lettre à Pauline Beyle, décembre 1800, dans Correspondance, édité par H. Martineau et V. Del Litto, Paris, Gallimard, coll. « Bibliothèque de la Pléiade », 1968, t. I, p. 14-15.

3. «J'ai adoré Saint-Simon en 1800 comme en 1836. Les épinards et Saint-Simon ont été mes seuls goûts durables" (Vie de Henry Brulard, dans CEuvres intimes, t. II, édité par V. Del Litto, Paris, Gallimard, coll. «Bibliothèque de la Pléiade », 1982, p. 931).

4. B. Diaz, "Mémoires du monde, mémoires de soi. Stendhal lecteur de Mémoires ", L'Année Stendhal, $\mathrm{n}^{\circ}$ 3, Paris, Klincksieck, 1999, p. 139.

5. Voir Journal (20 mars 1810), Euvres intimes, t. I [en abrégé : OI, I], édité par V. Del Litto, Paris, Gallimard, coll. «Bibliothèque de la Pléiade », 1981, p. 557.

6. Stendhal, Journaux et papiers. Volume I - 1797-1804, édité par C. Meynard, H. de Jacquelot et M.-

R. Corredor, Grenoble, Ellug, 2013, p. 143.

7. V. Del Litto avait partiellement publié L'Histoire de la guerre de Succession dans Mélanges I. Politique et Histoire, Genève, éd. Cercle du Bibliophile, t. 45, 1971. Acquis par la Bibliothèque municipale de Grenoble en 1996, un cahier inédit (62 pages rédigées) qui complète cette Histoire a été édité par C. Meynard: Histoire d'Espagne depuis la révolte du 28 avril 1699 jusqu'au Testament du 2 octobre 1700, Paris, Kimé, coll. « La Chasse au Snark », 2007.

8. V. Del Litto, ouvr. cité, p. 370.

9. M. Crouzet, Stendhal ou Monsieur Moi-Même, Paris, Flammarion, coll. «Grande Biographie », 1990, p. 127.

10. G. Rannaud, «Stendhal et la tentation de l'histoire », Romantisme, $n^{\circ} 107,2000$, p. 5-22.

11. La police et les censeurs pèsent sur tous les écrits (publiés, ou écrits en vue d'être publiés) de Stendhal. Voir Y. Ansel, Pour un autre Stendhal, Paris, Garnier, 2012, p. 32-37 ; 110-118 ; 124-136.

12. Voir Vie de Napoléon, dans Napoléon, éd. C. Mariette, Stock, 1998, p. 15 (début du chapitre 1).

13. Vie de Henry Brulard [en abrégé : VHB], Euvres intimes, t. II [en abrégé : OI, II], ouvr. cité, p. 540.

14. Journal (The history of his life, 1831), OI, II, ouvr. cité, p. 155.

15. Dans un compte rendu (février 1825) de l'ouvrage du comte de Ségur, Histoire de Napoléon et de la Grande Armée pendant la campagne de 1812, figure cette remarque: «Et pourtant, tout épouvantable qu'il est, ce récit est bien au-dessous de la réalité, qui fut marquée d'horreurs impossibles à présenter au public. » (Stendhal, Paris-Londres. Chroniques [en abrégé : PL], éd. R. Dénier, Paris, Stock, 1997, p. 271.)

16. « Il [Beyle] fit la campagne de Russie et se distingua par son sang-froid; il apprit au retour que cette retraite avait été une chose terrible. Cinq cent cinquante mille hommes passèrent le Niémen; cinquante mille, peut-être vingt-cinq mille le repassèrent" ("Notices autobiographiques ", 1837, OI, II, p. 977.)

17. Sur l'insistante présence et la portée de cette date récurrente, voir Y. Ansel, « Histoire de vie et sentiment de l'Histoire », dans Écrire l'histoire, nº 6, Marseille, Gaussen, 2010, p. 127-130.

18. « Notices autobiographiques » (6 janvier 1831), OI, II, p. 970-971.

19. J. Walch, Les maîtres de l'histoire 1815-1850, Genève, Slatkine, 1986, p. 31.

20. Voir Souvenirs d'égotisme, OI, II, p. 50 ; VHB, p. 729 et 951.

21. C'est dans l'Histoire de la peinture en Italie (1817) qu'apparaît, tout armée déjà, la conception stendhalienne d'un devenir historique non dialectisable (l'historien ne veut ni ne peut résoudre, "dépasser» les contradictions constatées; le réel n'est pas rationnel, il n'y a pas de raison cachée, pas de progrès sur toutes les lignes, non synchrones, divergentes, incompatibles, du temps). Sur tout ceci, voir Y. Ansel, Stendhal, le temps et l'Histoire, ouvr. cité, p. 168-186. 
22. La formule est de P. Barbéris, dans «Stendhal et la fascination du passé », Elseneur, $\mathrm{n}^{\circ} 2$, Presses universitaires de Caen, 1983, p. 7.

23. X. Bourdenet, "Ô dix-neuvième siècle! " Historicité du roman stendhalien : Armance, Le Rouge et le Noir, Lucien Leuwen (I, chap. 2) thèse dactylographiée, 2 vol., université de Besançon, 2004, p. 74-151.

24. Ibid., p. 76.

25. Voir Y. Ansel, «Stendhal et le pamphlet », Pour un autre Stendhal, ouvr. cité, p. 17-28.

26. Voir sur ce point Y. Ansel, «Le savant et la mort du dilettante », Stendhal Club, $\mathrm{n}^{\circ} 130$, Grenoble, 1991, p. 105-114.

27. Pour les détails de l'argumentation et les citations, voir Y. Ansel, Stendhal, le temps et l'Histoire, ouvr. cité, p. 302-310, et Pour un autre Stendhal, ouvr. cité, p. 165-182.

28. Une hérésie toujours hérétique, un paradoxe non encore devenu lieu commun, «truism». Dans la tribu des historiens, P. Veyne a naguère fait scandale en battant en brèche les illusions de la continuité historique, en insistant, à la suite de M. Foucault, sur la multiplicité des temps, sur les coupures, fractures et discontinuités chronologiques, sur les années-lumière qui nous séparent définitivement du monde aboli des Romains, de parfaits étrangers pour nous.

29. PL (mai 1829), p. 916.

30. Racine et Shakespeare II, dans Racine et Shakespeare, édité par R. Fayolle, Paris, GarnierFlammarion, 1970, p. 105.

31. Ibid., p. 107.

32. P. Barbéris, «Stendhal et la fascination du passé », art. cité, p. 5.

33. Pour le mécréant Stendhal, l'âme n'est nullement divine, «incréée », mais un produit culturel, historique : «L'âme des Italiens, pour lesquels peignit Michel-Ange, était formée par ces hasards heureux qui donnèrent au quinzième siècle presque toutes les qualités nécessaires pour les arts [...]» (Histoire de la peinture en Italie, édité par V. Del Litto, Paris, Gallimard, coll. « folio classique », 1996, p. 396) ; « Notre âme, quand elle entend gronder le tonnerre, n'est plus terrifiée. Pour beaucoup de gens ce son, souvent magnifique par sa plénitude, produit un effet musical. Le plus nuisible des athées, Benjamin Franklin, a eu l'impiété d'expliquer la foudre. Notre âme est donc bien différente de ce qu'était l'âme du Bourguignon en l'an 1200. Les églises romanes ou gothiques nous disent donc, exactement parlant, autre chose que ce qu'elles disaient à ces Barbares si sûrs de l'enfer.» (Mémoires d'un touriste, dans Voyages en France, édité par V. Del Litto, Paris, Gallimard, coll. «Bibliothèque de la Pléiade », 1992, p. 178.)

\section{RÉSUMÉS}

Entre Stendhal et l'histoire, c'est à fois une longue histoire d'amour (l'histoire est une passion) et un mariage de raison : l'histoire est un répertoire d'événements, de faits, d'actions, etc., qui peuvent être mobilisés, utilisés pour servir les causes les plus diverses. Fondamentalement, l'appel à l'histoire obéit à deux objectifs majeurs :

1) Mobiliser les époques, les événements passés pour critiquer le siècle, rapetisser les temps modernes, disqualifier les sociétés trop « perfectionnées»;

2) Prendre acte que l'homme est un "animal historique ", et tirer toutes les conséquences (sociales, politiques, psychologiques, philosophiques, artistiques, éthiques, etc.) de cette idée neuve. 
Between Stendhal and History, there is both a long-standing love story (History is a passion) and a marriage of convenience: History is a pool of events, facts, actions, etc., which can be mobilized, used to serve the most various causes. As Bayle or Voltaire did before him, Stendhal exploits History, using it as a formidable, efficient weapon. Fundamentally, referring to History serves two significant aims:

1) first, to mobilize periods and former events in order to criticize the 19th century, disparage modern times and disqualify "oversophisticated" societies;

2) it also implies that a person is a "historical animal", while drawing all the social, political, psychological, philosophic, artistic, ethical conclusions from this new idea.

\section{AUTEUR}

\section{YVES ANSEL}

Université de Nantes.

Yves Ansel est professeur de littérature française des $\mathrm{XIX}^{\mathrm{e}}$ et $\mathrm{XX}^{\mathrm{e}}$ siècles à l'université de Nantes. Il a notamment publié des études sur Sartre, Nizan, Vian et Camus (Albert Camus totem et tabou. Politique de la postérité, 2012). Sur Stendhal, il a écrit une cinquantaine d'articles, plusieurs ouvrages (dont Pour un autre Stendhal, Garnier, 2012), co-dirigé le Dictionnaire de Stendhal (Champion, 2003) et les Euvres romanesques complètes (Gallimard, Bibliothèque de la Pléiade : 2005, 2007, 2014). 